\title{
Single-shot spatial-temporal electric field measurement of intense terahertz pulses from coherent transition radiation
}

\author{
Qili Tian, Yingchao Du, Hanxun Xu, Yifan Liang, Yi Wang, Yuemei Tan, Lixin Yan๑, \\ Renkai Li, Cheng Cheng, Wenhui Huang, and Chuanxiang Tang \\ Department of Engineering Physics, Tsinghua University, Beijing 100084, China \\ Key Laboratory of Particle and Radiation Imaging, Tsinghua University, \\ Ministry of Education, Beijing 100084, China
}

(Received 28 May 2020; accepted 2 October 2020; published 15 October 2020)

\begin{abstract}
Coherent transition radiation (CTR) is a widely used approach to characterize the electron beam in accelerator science and technology. Characterization of the CTR with an efficient, versatile and compact setup is in considerable demand for a wide range of applications such as free electron lasers, electron storage rings and other related scientific researches. In this article, we report on spatial-temporal electric field measurements of CTR using the single-shot electro-optic spatial decoding method. A quasi-half-cycle temporal waveform of the THz pulse with peak electric field strength of $\sim 42 \mathrm{MV} / \mathrm{m}$ has been measured. A symmetrical field profile across the transverse center and the distorted wave front of CTR THz pulses near the focal plane were demonstrated. Moreover, timing jitters of the ultrashort electron beams are also monitored via this method with tens of femtosecond accuracy. This comprehensive method provides the temporal waveform and the one-dimensional spatial imaging of THz fields simultaneously, showing it has the potential for single-shot diagnostics of ultrashort electron beams.
\end{abstract}

DOI: $10.1103 /$ PhysRevAccelBeams.23.102802

\section{INTRODUCTION}

Recently, intense THz radiations with peak electric field strength exceeding $100 \mathrm{MV} / \mathrm{m}$ have attracted significant interest due to increasing applications in condensed matter [1,2] and accelerator physics [3-7]. The most prominent approaches for generating high peak field $\mathrm{THz}$ radiation are optical rectification with ultrashort laser pulses [8-11] and coherent transition radiation (CTR) based on relativistic electron beams [12-21]. CTR is generated by ultrashort electron bunches interacting with a metal screen and the total radiated power scales with the square of the electron numbers $[13,14]$. Intense $\mathrm{THz}$ radiation with field amplitude up to multi-GV/m and mJ-level pulse energies have been demonstrated by CTR [18-21], which may find applications in $\mathrm{THz}$ acceleration exploration for its distinguished features of radially polarized electric field and intrinsically self-synchronized with the electron beams from the accelerator [22].

Meanwhile, CTR carries the longitudinal distribution information of the electron bunches [14,23]. Temporal

\footnotetext{
*yanlx@mail.tsinghua.edu.cn
}

Published by the American Physical Society under the terms of the Creative Commons Attribution 4.0 International license. Further distribution of this work must maintain attribution to the author(s) and the published article's title, journal citation, and DOI. diagnostics of electron bunches in accelerator facilities [24-27] and laser-plasma wakefield accelerations [28,29] have been performed by characterizing CTR THz radiation from the electron beams. One of the main approaches for measuring CTR is THz autocorrelation, including MartinPuplett interferometer [27] and Michelson interferometer $[19,25,26,30]$, which measure the autocorrelation curve instead of the temporal waveform. Another approach is the electro-optic (EO) sampling technique, in which a synchronized ultrashort laser beam is applied to scan the $\mathrm{THz}$ pulse inside the EO crystal with an optical delay line [12]. However, this scanning probe technique suffers the shot-to-shot instability of $\mathrm{THz}$ pulses and the timing jitter between the probe laser and the electron beam. Alternatively, the single-shot detection technique is highly demanded especially for low repetition rate accelerators. Moreover, the single-shot EO method can also be applied to monitor the timing jitter between electron bunches and probe laser which is essential for beam feedback control [31-33].

Extensive research efforts have been devoted to singleshot EO techniques for temporal profile characterization of relativistic Coulomb fields [34-39], coherent synchrotron radiation [40,41] and coherent diffraction radiation [42]. In the single-shot detection of $\mathrm{THz}$ temporal waveform based on CTR, the EO spectral decoding method was first performed at FLASH [32]. The temporal resolution is limited by the spectral bandwidth of the picosecond chirped optical probe pulse, where frequency mixing of the spectral 
components of $\mathrm{THz}$ pulse with the probe laser spectrum broadens and distorts the signal [43]. To overcome this limitation, the EO temporal decoding method [44] and the temporal electric field cross correlation scheme [45] have been proposed. Nevertheless, only a portion of the radially polarized cone of $\mathrm{THz}$ emission was measured in these experiments.

In the present study, we directly characterized the temporal profile and 1D spatial electric field distribution of radially polarized $\mathrm{THz}$ pulses by the $\mathrm{EO}$ spatial decoding method with high time resolution in single shot. The symmetrical field profile across the transverse center and the distorted wave front of $\mathrm{THz}$ pulses near the focal plane were investigated. Intense $\mathrm{THz}$ radiation with a peak field of $\sim 42 \mathrm{MV} / \mathrm{m}$ was measured with $30 \mathrm{MeV}$ ultrashort electron beams at the Tsinghua Thomson scattering $\mathrm{x}$ ray source (TTX) [46]. With this scheme, the relative timing jitter between the electron beam and the probe laser was monitored with femtosecond accuracy.

\section{THEORETICAL DESCRIPTION}

As relativistic charged particles cross the boundary between two media of different dielectric constants, the electromagnetic field of the charged particles excites current at the boundary, thus generating transition radiation $[23,32]$. The physical nature of the transition radiation can be theoretically investigated by the virtual photon method with arbitrary screen size at a certain observation distance $[14,47]$. For relativistic electron bunches, when the wavelength of the transition radiation is longer than the bunch length, electrons in the bunch radiate coherently, making the radiation intensity grow quadratically with the bunch charge [48]. For hundreds-of-femtosecond electron bunches the CTR spectrum lies in the $\mathrm{THz}$ regime.

The EO sampling technique utilizes the field-induced birefringence effect in the EO crystal to measure the electric field of the $\mathrm{THz}$ pulse. Due to the THz-field-induced birefringence, the linearly polarized probe laser propagating in the EO crystal will experience a relative phase retardation between the two polarization components, which is proportional to the $\mathrm{THz}$ electric field [49]. For instance, a ZnTe crystal used in EO experiments is cut in the (110) plane and a radially polarized THz pulse incidents perpendicular to this plane. As the probe laser beam incidents into the $\mathrm{ZnTe}$ crystal at an angle of $\theta$ relative to the surface normal, the relative phase retardation in the crystal is given by

$$
\Gamma=\frac{2 \pi L n_{0}^{3} r_{41} E_{\mathrm{THz}}}{\lambda_{0} \sqrt{\eta} \cos \theta}
$$

assuming the linear laser polarization in the (110) plane parallel to the $[-1,1,0]$ axis of the crystal, where $E_{\mathrm{THz}}$ is the integral projection of the radial electric field on the $[-1,1,0]$ axis, $\lambda_{0}=800 \mathrm{~nm}$ is the central wavelength of probe laser, $n_{0}=2.85$ is the refractive index of the crystal at wavelength $\lambda_{0}$, L is the crystal thickness, $r_{41} \simeq 4 \times 10^{-12} \mathrm{mV}^{-1}$ is the EO coefficient of $\mathrm{ZnTe}$, $\eta \simeq 6$ is the $\mathrm{THz}$ transmission loss induced by the $\mathrm{ZnTe}$ crystal dispersion and velocity mismatch between the laser and $\mathrm{THz}$ pulse $[28,50]$.

For measuring the relative phase retardation, an analyzing polarizer is placed after the EO crystal to separate the orthogonal electric field components of the modulated probe laser, where temporally local phase retardation is converted to measurable intensity modulation. In singleshot EO detection, only the vertical polarization component of the probe laser was measured [39]. Normally, a quarterwave plate $(\lambda / 4$ plate) is inserted between the EO crystal and the analyzing polarizer to yield a circular polarization state in the absence of $\mathrm{THz}$ field. As a horizontally polarized probe laser $I_{\mathrm{pr}}$ passing through the EO crystal, the modulated vertical polarization component is detected, which gives [50]

$$
I_{\mathrm{dec}}(\tau)=\frac{I_{\mathrm{pr}}}{2}[1-\sin \Gamma(\tau)]
$$

The detection intensity $I_{\mathrm{dec}}(\tau)$ is a function of $E_{\mathrm{THz}}$, and with a background $I_{\mathrm{pr}} / 2$.

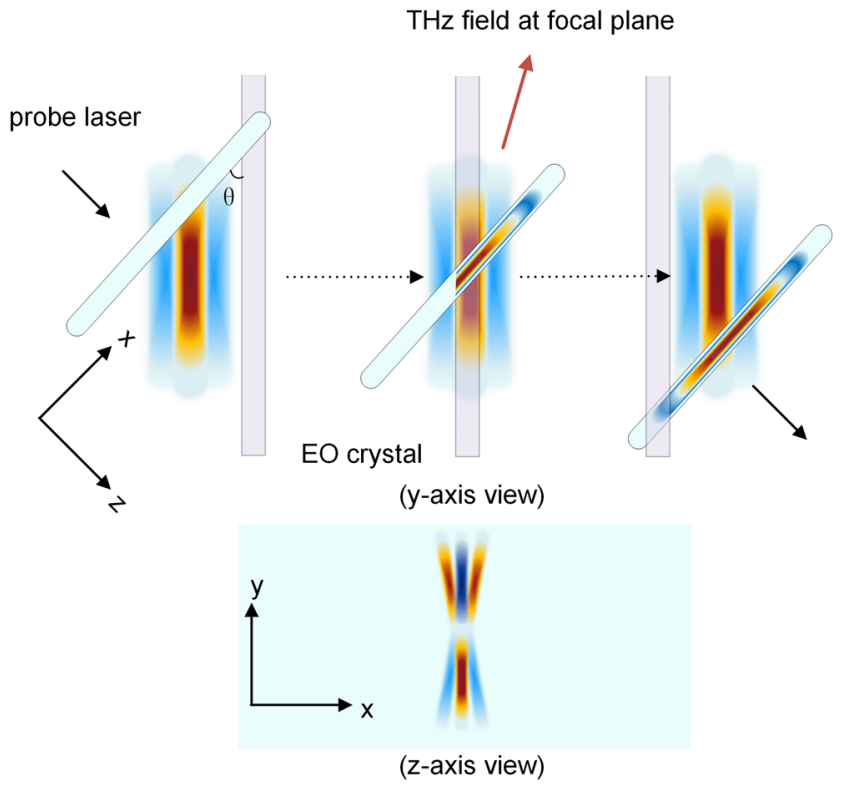

FIG. 1. Schematic description of the EO spatial decoding method. The y-axis view shows three instants during the course of the measurement. The lavender object represents the EO crystal. The colorful object represents the $\mathrm{THz}$ electric field distribution at the focal plane. The aqua object represents a probe laser pulse passing through the EO crystal with an angle $\theta$ relative to the $\mathrm{THz}$ pulse travel direction. Hence, a different cross section of the probe laser was modulated by the $\mathrm{THz}$ field with different arrival time in the EO crystal. The $\mathrm{z}$-axis view shows the modulated transverse spatial profile of the probe beam. 
Different from the collinear detection geometry, the schematic of EO spatial decoding detection is shown in Fig. 1. A linearly polarized ultrashort probe pulse and a radially polarized $\mathrm{THz}$ pulse interact in the $\mathrm{EO}$ crystal at an angle $\theta$, different spatial components of the probe laser pass through the crystal at different times and acquire a different elliptical polarization. Therefore, spatial-temporal field distribution of the $\mathrm{THz}$ pulse is mapped to the transverse spatial profile of the probe beam, where the z-axis represents the direction of probe laser propagation. The modulated polarization state of the probe beam can be detected by an area array charge-couple device (CCD) camera via orthogonal polarization detection geometry. In the transverse spatial profile of the probe beam (z-axis view in Fig. 1), the $x$ coordinate maps the temporal profile while another dimension (y coordinate) is used to record 1D spatial distribution of the $\mathrm{THz}$ field. The modulated amplitude of the probe laser is applied for $\mathrm{THz}$ field strength measurement. The mapped temporal pulse shape is symmetrical in the 1D spatial direction but with opposite amplitude due to the rotational symmetry of the radial electric field polarization. The symmetrical spatialtemporal electric field profile of the radially polarized $\mathrm{THz}$ pulse can be measured by this method with a single shot manner.

The linear temporal mapping relationship between the $\mathrm{THz}$ field coordinate $t_{i}$ and the probe laser transverse spatial coordinate $x_{i}$ writes as [51]

$$
t_{i}=\frac{x_{i} \tan \theta}{c},
$$

where $\theta$ is the relative angle between the THz pulse and the probe laser, and $\mathrm{c}$ is the speed of light in vacuum. As it can be seen, the total time window depends on the angle $\theta$ and the effective transverse length of the probe laser beam. A time window of more than a dozen of picoseconds is possible for measuring multicycle $\mathrm{THz}$ pulses.

\section{EXPERIMENTAL SETUP}

The experiment with intense $\mathrm{CTR} \mathrm{THz}$ radiation was performed at TTX, which includes the electron beam line, the ultraviolet (UV) driving laser and the infrared (IR) scattering laser system. The schematic of the experiment is presented in Fig. 2. A flattop 9 ps (FWHM) UV driving laser for the photocathode $\mathrm{rf}$ gun is created by pulse stacking using birefringent $\alpha-\mathrm{BBO}$ crystal serials [52]. A $100 \mu \mathrm{J}$ probe laser is separated from the $30 \mathrm{TW} / 30 \mathrm{fs}$ Ti:sapphire IR laser system for EO detection. Both laser systems are operated at a repetition rate of $10 \mathrm{~Hz}$, and phase locked to a $2856 \mathrm{MHz}$ master clock signal. In the beam line, the beam charge can be varied from a few $\mathrm{pC}$ up to $\sim 1 \mathrm{nC}$ by tuning the UV pulse energy. The electron bunch is accelerated by a $3 \mathrm{~m}$ SLAC-type traveling wave accelerating tube to $45 \mathrm{MeV}$ at the maximum acceleration phase. A magnetic chicane is used to compress the bunch to hundreds of femtoseconds for CTR generation. More details about the system could be found in Ref. [53].

The setup for the intense CTR THz generation and the EO spatial decoding detection is shown in Fig. 3. An aluminized mirror with 2-inch diameter oriented at 45 degrees with respect to the beam line was installed to generate CTR. The electron beam was accelerated at $-40^{\circ} \mathrm{rf}$ phase for later bunch compression in the magnetic chicane, resulting in a beam energy of $30 \mathrm{MeV}$. The backward radiation of CTR was collected by an off-axis parabolic mirror (OAP1) with $101.8 \mathrm{~mm}$ focal length. A silver mirror was used to transport $\mathrm{THz}$ radiation out of the vacuum chamber. The radiation passed through a 2 -inchdiameter, 2-mm-thick TPX window and was focused by OAP2, which has the same parameters as OAP1. The pulse energy and transverse distributions of $\mathrm{THz}$ radiation were measured near the focal plane. A 300- $\mu \mathrm{m}$-thick, $10 \times 10-\mathrm{mm}^{2}$-size ZnTe crystal was placed in the focal plane of THz pulses. The ZnTe crystal was cut in the (110) plane, and its $[-1,1,0]$ axis in the horizontal direction could be slightly rotated to optimize the EO signal.

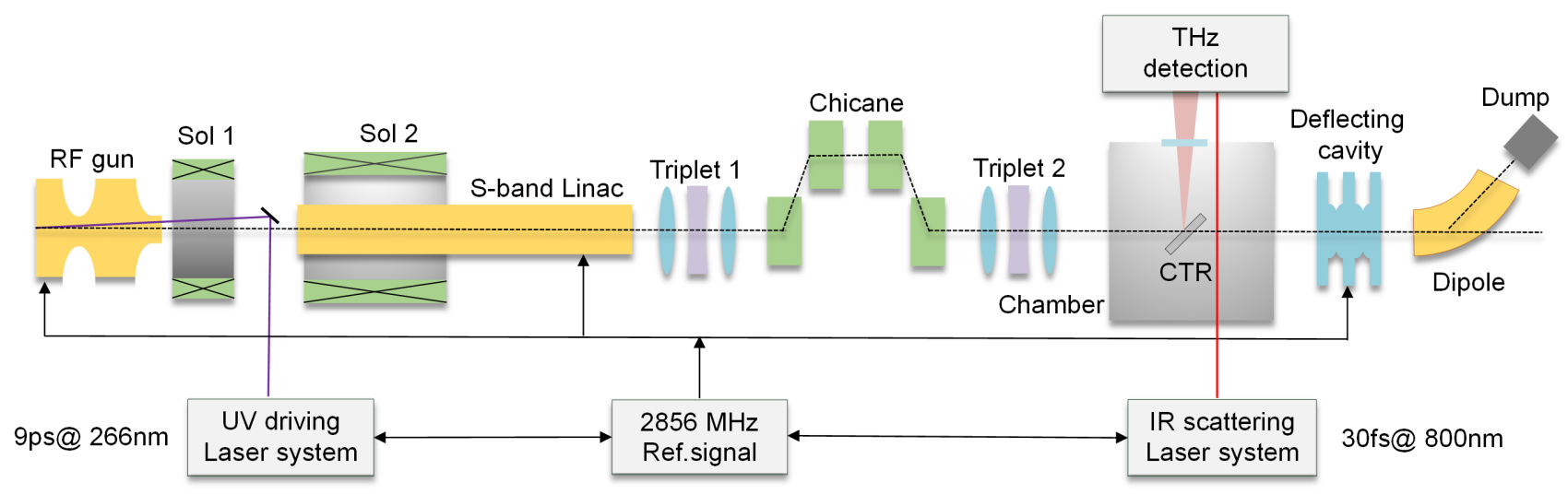

FIG. 2. Schematic layout of the beam line for CTR. 


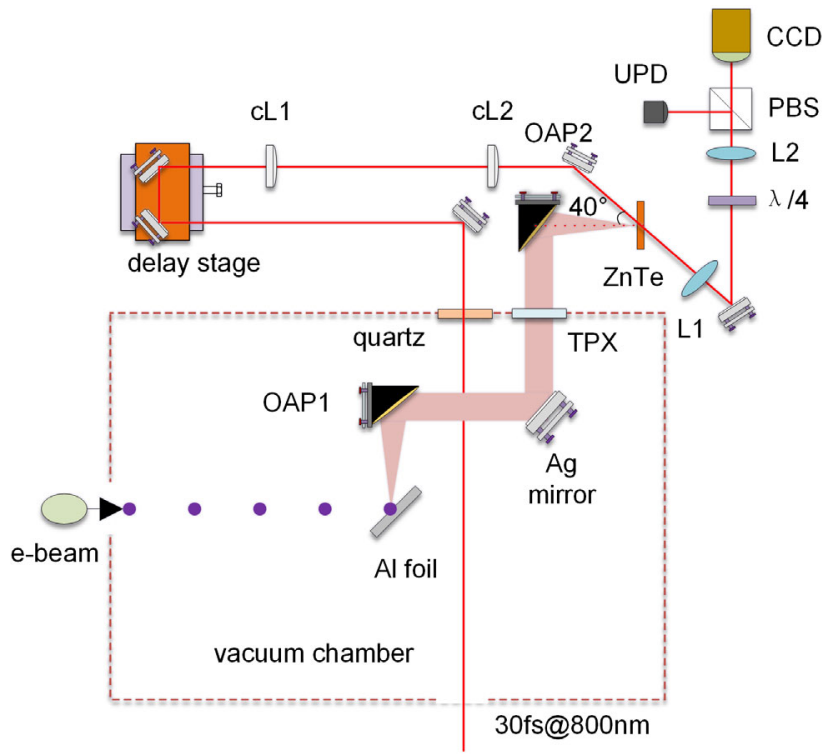

FIG. 3. Schematic of the EO spatial decoding detection. The radially polarized $\mathrm{THz}$ pulse incidents into the $\mathrm{ZnTe}$ crystal along the normal to the (110) plane and the horizontally polarized probe laser incidents into the $\mathrm{ZnTe}$ crystal at an angle of $40^{\circ}$ relative to normal of the crystal surface. OAP: $90^{\circ}$ off-axis parabolic mirror; TPX: poly(4-methyl-1-pentene); cL: cylindrical lens; UPD: ultrafast photodiode; PBS: polarized beam splitter.

After passing through a 3-mm-thick quartz window, the probe laser obliquely incidents into the ZnTe crystal. The relative angle between probe laser and $\mathrm{THz}$ pulse was set to be $40^{\circ}$. A motorized delay stage was used to configure the time delay between the probe laser and the $\mathrm{THz}$ pulse. To increase the time resolution of EO detection, the probe laser was reshaped to $3 \mathrm{~mm}$ in vertical and $15 \mathrm{~mm}$ in horizontal by two cylindrical lenses with focal length $f_{1}=250 \mathrm{~mm}$ and $f_{2}=50 \mathrm{~mm}$. The pulse length of the probe laser was broadened to $57 \mathrm{fs}$ at the EO crystal due to dispersion of the quartz window and the cylindrical lens. The modulated probe laser was transferred to the orthogonal polarization configuration by a pair of lenses $(f=150 \mathrm{~mm})$ through imaging relay. A $\lambda / 4$ wave plate was used to yield a circular polarization state in the absence of the $\mathrm{THz}$ field. The vertical polarization light was split by a polarized beam splitter (PBS) and transported to a CCD with $\sim 5 \mu \mathrm{m}$ pixel pitch.

\section{RESULTS AND DISCUSSION}

\section{A. EO spatial decoding signal and temporal mapping relationship calibration}

The typical measurement results by single-shot EO spatial decoding technique are shown in Fig. 4. The bunch charge is $200 \mathrm{pC}$ and a $\mathrm{THz}$ attenuator with $10 \%$ transmittance was used to avoid over-rotation of the phase modulation [54]. Unlike the crossed polarization geometry with near zero background [55], an appropriate background

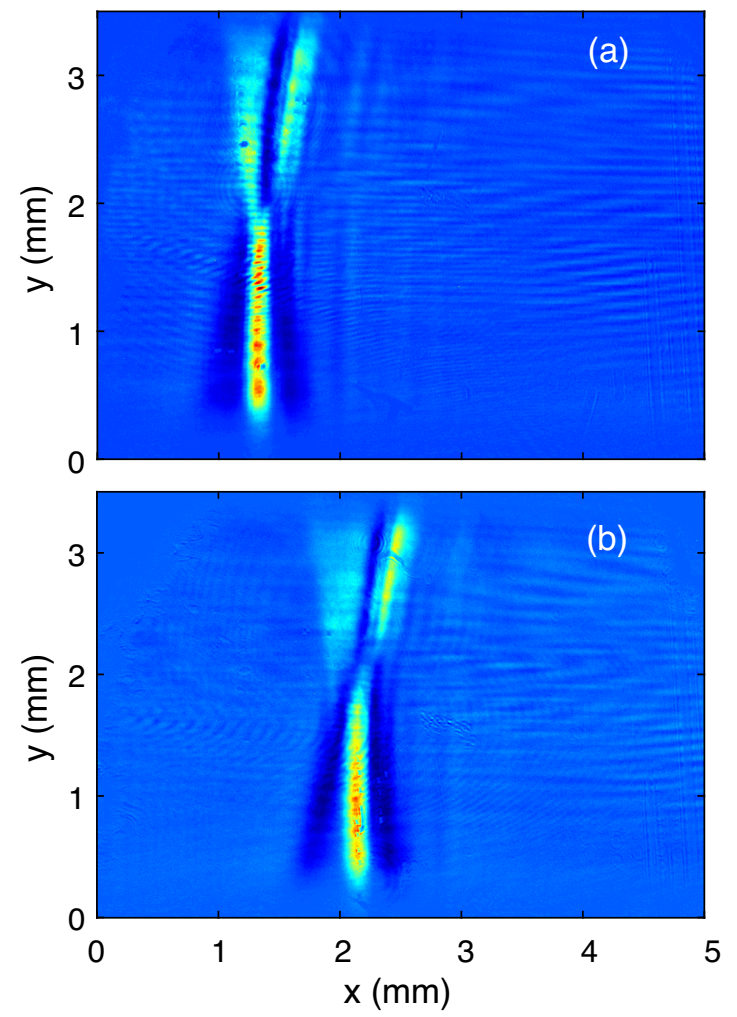

FIG. 4. The typical measurement results by the single-shot EO spatial decoding method near the focal plane of $\mathrm{THz}$ pulse. (a) THz pulse synchronization with the probe laser; (b) 2 ps delay between $\mathrm{THz}$ pules and probe laser.

is required for measuring bipolar electric fields. 200 shots were taken with the same laser and CCD settings for laser background subtraction, some residual noise remained in the EO signal due to the probe laser beam pointing instabilities and mechanical vibrations of the setup.

Figure 4(a) is the uncalibrated spatial-temporal electric field distributions of radially polarized $\mathrm{THz}$ pulse near the focal plane, where the $\mathrm{x}$ coordinate is related to the temporal waveform of the field while the $y$ coordinate related to $1 \mathrm{D}$ spatial field distribution. The range of the measured field is related to the effective transverse size of the probe laser $(3 \times 5 \mathrm{~mm}$, where the horizontal direction of the probe laser is cut off by the mount of ZnTe crystal). The measured $\mathrm{THz}$ field distribution is antisymmetric with respect to its center, which contributes unambiguous evidence of the radial polarization of the CTR pulse. The oscillations in the tailing part of the EO signal might be caused by dispersion inside the ZnTe crystal and group velocity mismatch between the probe laser and the $\mathrm{THz}$ pulse.

It is generally known that the Gaussian beam has a planar wave front at the waist. As expected, the wave front of the THz pulse at the bottom of Fig. 4(a) is nearly flat. But at the top part of 4(a), the EO signal showed a curved structure. This suggested that the position of the ZnTe crystal was 
slightly offset from the $\mathrm{THz}$ focal plane and the wave front was deviated from the plane wave. If the arrival time of the probe laser was relatively delayed for $2 \mathrm{ps}$, as shown in Fig. 4(b), the observed wave front of the $\mathrm{THz}$ pulse is deviated more from plane wave. This means that the more relative distances between the field spatial position and the center of the focus, the more obvious the bending phenomenon of the wave front. The bending direction is correlated to the deviation of the front and back focal plane. Hence, the EO spatial decoding technique can be an effective method for $\mathrm{THz}$ wave front diagnostics. Corresponding to a high-amplitude central pulse of one polarity, the two longer but weaker lobes of opposite polarity have larger divergent angles, which is consistent with the propagation characteristics of $\mathrm{THz}$ pulses in Refs. [18,56].

To calibrate the temporal mapping relationship, we captured part of the spatial distribution at seven different probe delays. By moving the delay stage with a step of $1 \mathrm{ps,}$ the position of the EO signal in the laser profile also moves for a certain distance. For data management, we averaged the peak positions of 200 consecutive EO signals at each probe delay to reduce the influence of the time jitter between the electron beam and the probe laser. Measured data of temporal mapping relationship, linear fitting results and numerical calculations by Eq. (3) are shown in Fig. 5. The mean positions of 200 shots are denoted by green dots and standard deviations are denoted by error bars. The red line is the linear fitting of experimental data with a calibration factor of $12.8 \mathrm{fs} /$ pixel, corresponding to a crossing angle of $38^{\circ}$. The blue line shows the numerical calculations of a planar wave from Eq. (3), the calibration factor of $13.9 \mathrm{fs} /$ pixel is obtained with $\theta=40^{\circ}, \Delta x=5 \mu \mathrm{m}$, which is slightly larger than the

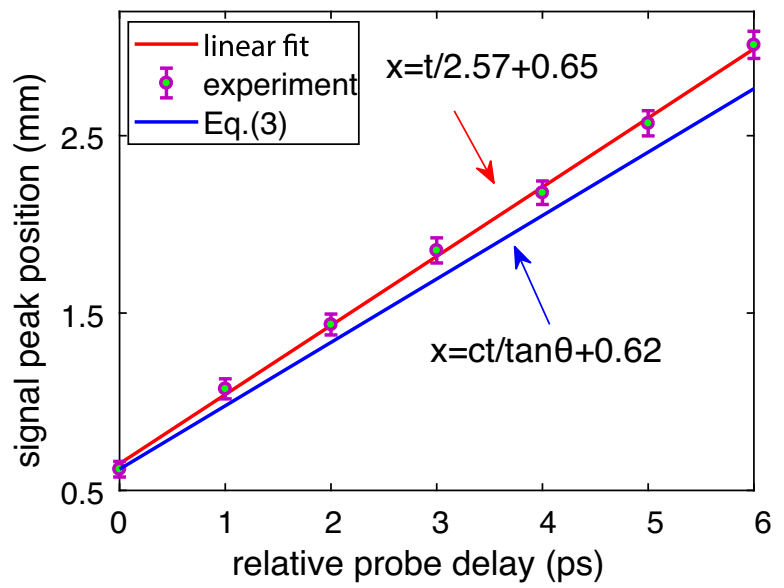

FIG. 5. Measurements and theoretical calculations of the temporal mapping relationship. The mean positions are denoted by green dots and standard deviations are denoted by error bars. The red solid line denotes a linear fitting of the experimental results, while the blue solid line illustrates the numerical calculations by Eq. (3). experimental data. It might be caused by the deviation of the angle $\theta$ in the experimental configuration and the bending wave front of the $\mathrm{THz}$ pulse in Fig. 4. Moreover, the arrival time jitter of the electron bunches would also contribute to the deviation of the calibration factor. This effect can be mitigated by a method of two gate probe pulses with variable delay proposed by Steffen et al. [39].

\section{B. Temporal waveform and spectrum of $\mathrm{THz}$ pulse}

The calibrated spatial-temporal electric field characteristics of $\mathrm{THz}$ radiation near the focal plane are shown in Fig. 6(a), where the field pattern exhibits a central hole due to the radial polarization feature of the CTR electric field. The temporal $\mathrm{THz}$ waveform varies along the 1D spatial
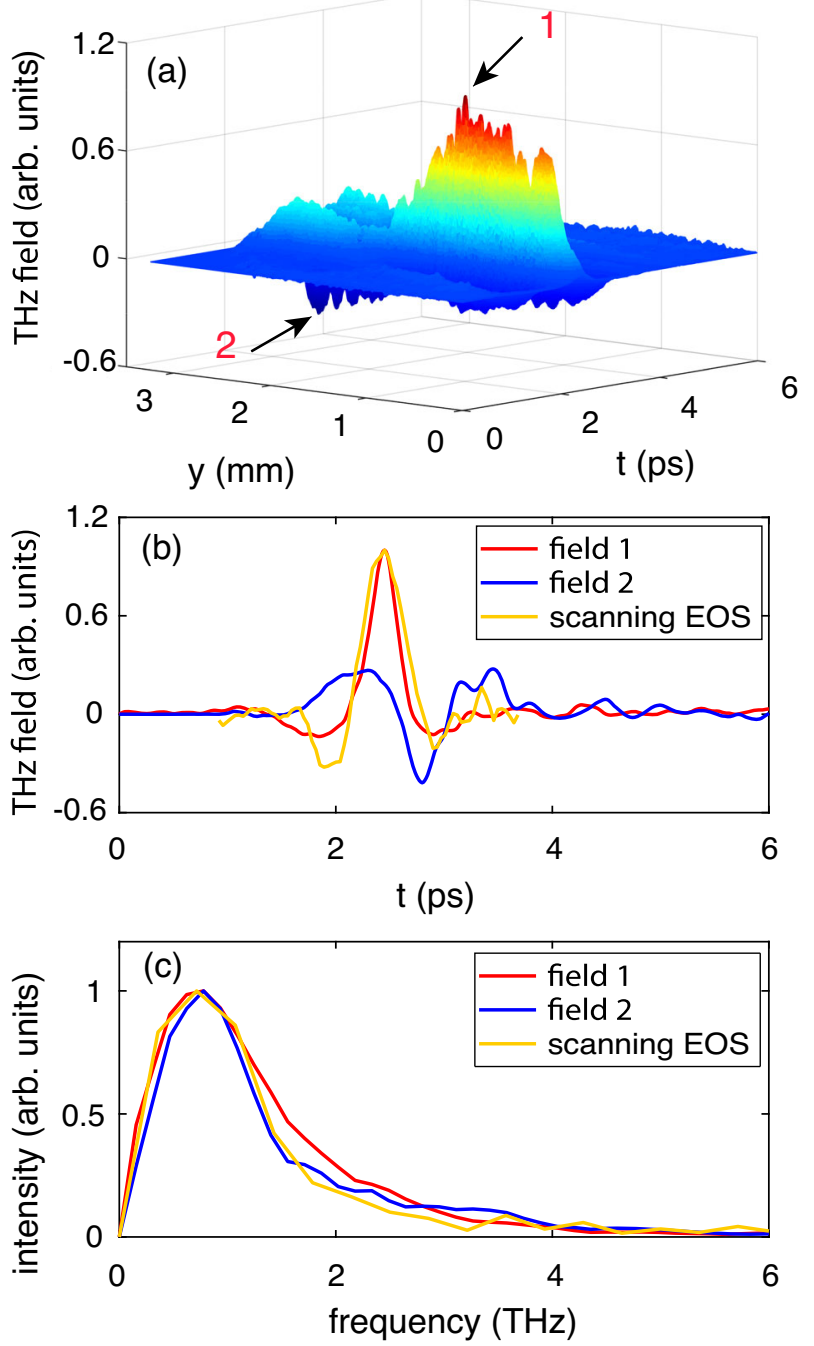

FIG. 6. (a) The spatial-temporal electric field distribution of $\mathrm{THz}$ radiation measured by the EO spatial decoding method at the focal plane. The temporal waveforms (b) and spectrum (c) of the $\mathrm{THz}$ field at position 1 (red solid line, FWHM of $310 \mathrm{fs}$ ), position 2 (blue solid line, FWHM of $380 \mathrm{fs}$ ) and scanning EOS (yellow solid line, FWHM of $360 \mathrm{fs}$ ). 
position (y-axis), where the pulse duration increases with the position of the field away from the central hole. The field strength at the central hole is approximately zero, and there are two peaks (position 1 and position 2) with opposite polarity along the $1 \mathrm{D}$ spatial directions. The spatial positions of peaks 1 and 2 are found roughly 0.8 and $0.9 \mathrm{~mm}$ from the central hole, respectively. The layered structures of the field strength in the y-axis direction are due to the optical inhomogeneity of the ZnTe crystal and the nonuniform transverse intensity distribution of the probe laser. A probe laser beam with uniform spatial distributions and a high quality ZnTe crystal would provide better spatial resolution. The temporal $\mathrm{THz}$ waveforms at position 1 (red solid line) and position 2 (blue solid line) are shown in Fig. 6(b). As a comparison, Fig. 6(b) also shows a THz signal measured by the electro-optic sampling (EOS) method with a scanning delay line (yellow solid line). The THz waveforms measured by the two methods are well consistent and both have typical quasi-half-cycle shape, which is caused by the diffraction of low frequency $\mathrm{THz}$ propagation and finite transverse dimension of the CTR screen [56].

The peak phase retardation induced by the $\mathrm{THz}$ pulse at position 1 was measured to be $\sim 1.5 \mathrm{rad}$, which is close to the detection limit of EO sampling detection. From these measurement results, the peak electric field strength is calculated to be $\sim 42 \mathrm{MV} / \mathrm{m}$ without the $\mathrm{THz}$ attenuators by Eq. (1). The pulse durations at positions 1 and 2 and EOS measurement were measured to be $\sim 310, \sim 380$ and $\sim 360 \mathrm{fs}$, respectively. The deviation of the peak position in time (positions 1 and 2) is mainly attributed to the curved wave front of THz pulse, which has been shown in the top parts of Fig. 4(a). By using the Fourier transform, we also obtained the corresponding spectrum of the $\mathrm{THz}$ field for the two techniques. As shown in Fig. 6(c), both have similar spectrum distribution ranged in $0.1-3 \mathrm{THz}$ centered at $\sim 0.78 \mathrm{THz}$.

It is interesting to note in Fig. 6(b) that the amplitude of the electric field at position 1 is almost twice of the field at position 2 . We consider that one of the reasons might be the asymmetry of THz spots that induced the difference of field strength at spatial distribution. The transverse profile of $\mathrm{THz}$ radiation was measured by a $384 \times 288$ pixel uncooled microbolometer focal plane array $\mathrm{THz}$ camera with $35 \mu \mathrm{m}$ pixel pitch from INO Corporation (model MICROXCAM-384i-THz). The spatial profile at the focal plane of a $90^{\circ}$ silver-coated off-axis parabolic mirror (OAP2) was taken and shown in Fig. 7(a). The minimum in the center of the focus shows the radial polarization characteristics of the CTR radiation. In Fig. 7(b), the radius in the $x$ direction (horizontal) and the y directions (vertical) are 1.6 and $1.5 \mathrm{~mm}$ (full width at $1 / e^{2}$ ), respectively. The measurement shows obvious asymmetry along horizontal distribution, which is attributed to the $45^{\circ}$ angle of inclination of the radiation screen with respect to the electron beam axis [42].

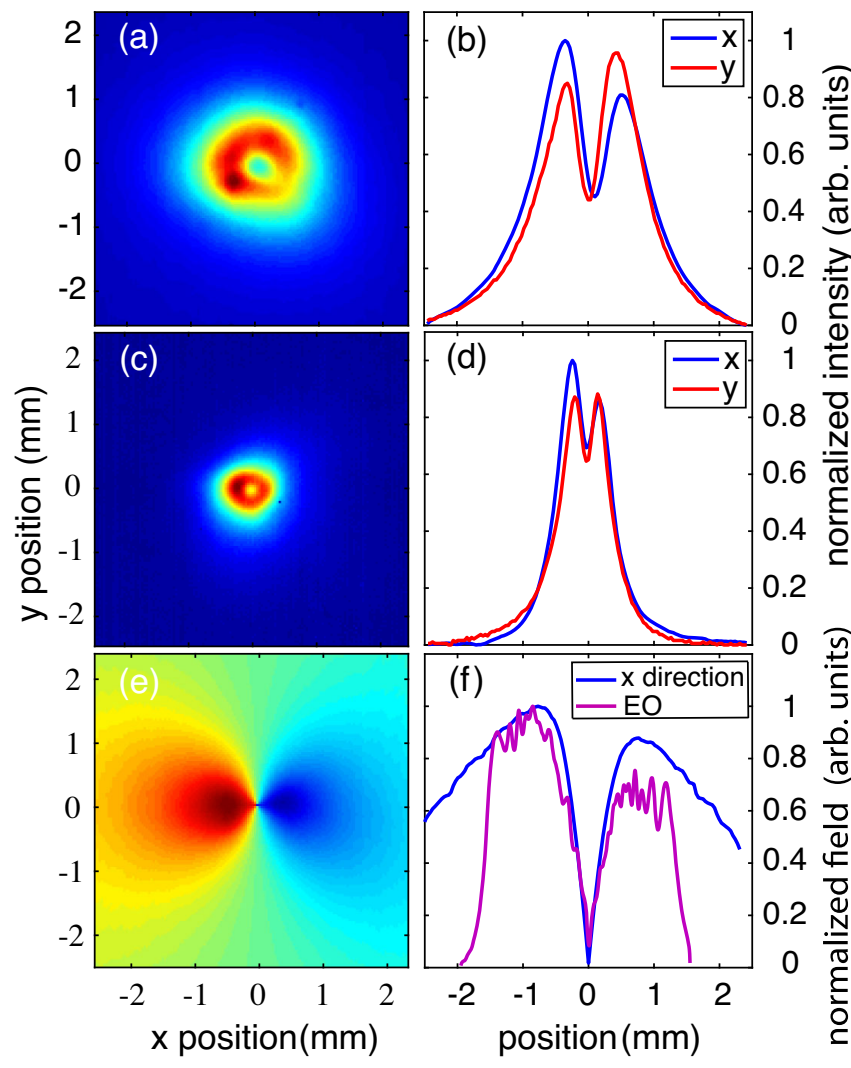

FIG. 7. The spatial distribution of the THz focal spot with (a) OAP2 $(\mathrm{D}=50.8 \mathrm{~mm}, \mathrm{f}=101.6 \mathrm{~mm})$ and (c) OAP (D $=50.8 \mathrm{~mm}, \mathrm{f}=50.8 \mathrm{~mm}$ ). (b), (d) Cross-section intensity profiles of the THz image in (a) and (c). (e) The field distribution convert from intensity distribution of (a). (f) The projection of the $\mathrm{THz}$ field strength (absolute value of amplitude) in the horizontal direction of the spatial distribution.

Asymmetry of vertical distribution may be related to the propagation of $\mathrm{THz}$ electromagnetic radiation [14] and aberration introduced by OAP2 focusing.

In our experimental configuration, the $[-110]$ axis of the ZnTe crystal is in the horizontal direction and the probe laser beam is polarized horizontally. Therefore, the 1D spatial measurement is the projection of the $\mathrm{THz}$ field in the horizontal direction. For a comparison of the 1D spatial measurement between the EO spatial decoding method and the $\mathrm{THz}$ camera, we converted the intensity distribution in Fig. 7(a) to the electric field distribution and performed an integral projection in the horizontal direction. As shown in Fig. 7(e), the relative field strength is the value of the horizontal component of the square root of the $\mathrm{THz}$ spot [Fig. 7(a)] intensity distribution. The color represents the different directions of the $\mathrm{THz}$ field. Figure 7(f) shows the projection of the $\mathrm{THz}$ field strength (absolute value of amplitude) in the horizontal direction of the spatial distribution. The 1D spatial measurements of EO spatial decoding (magenta solid line) reasonably agree with the spatial distribution acquired in a single-shot by a $\mathrm{THz}$ 
camera (blue solid line). The spatial distance between two field peaks is $1.55 \mathrm{~mm}$, which approximately equals the radius of the $\mathrm{THz}$ spot in the horizontal direction (full width at $\left.1 / e^{2}\right)$. The amplitudes of the electric field drop sharply to zero at the two sides of the horizontal direction, which are attributed to the effective transverse size of probe laser beam (3 $\mathrm{mm}$ in vertical) in EO spatial decoding detection. As it can be seen in Fig. 7(f), the amplitude ratio of two peaks measured by a $\mathrm{THz}$ camera and the EO spatial decoding method are $\sim 1.14$ and $\sim 1.33$, respectively. The possible reasons might originate from the angular deviation of the $\lambda / 4$ wave plate set up in the experimental configuration, the intrinsic birefringence and optical inhomogeneity of the ZnTe crystal, and more work needs to be done to make it clear.

For the $200 \mathrm{pC}$ electron bunch, the $\mathrm{THz}$ pulse energy was measured to be $\sim 10 \mu \mathrm{J}$ at the focus by a calibrated Golay cell from TYDEX [57]. Meanwhile, the THz peak electric field strength of $\sim 57 \mathrm{MV} / \mathrm{m}$ was calculated from the measured pulse energy, duration and focal spot size, which was in good agreement with the measurement in EO spatial decoding detection. For the $1 \mathrm{nC}$ electron bunch, the measured pulse energy exceeded $\sim 160 \mu \mathrm{J}$ with a pulse duration of $320 \mathrm{fs}$. To achieve higher electric field, an offaxis parabolic mirror (OPA) with shorter focal length of $50.8 \mathrm{~mm}$ was used instead of OAP2, and the measured focal spot intensity distribution is shown in Fig. 7(c). In Fig. 7(d), the measured $\mathrm{THz}$ spot radius (full width at $1 / e^{2}$ ) are $0.8 \mathrm{~mm}$ in the horizontal and $0.78 \mathrm{~mm}$ in the vertical directions. The peak electric field strength up to $\sim 450 \mathrm{MV} / \mathrm{m}$ was obtained when a shorter focal length OPA [Fig. 7(c)] and $1 \mathrm{nC}$ electron bunch were used in the experiment.

\section{Arrival time measurements}

Apart from spatial-temporal field characterization of $\mathrm{THz}$ radiation, the single-shot EO spatial decoding technique can also be applied to measure the timing jitter of the $\mathrm{THz}$ pulse relative to the probe laser. While the $\mathrm{THz}$ radiation is synchronized with the electron bunch, such measurement results can be used for monitoring the arrival timing of electron bunches. Figure 8 shows the measured $\mathrm{THz}$ field distribution of five consecutive bunches at one electric field direction (such as the bottom part of Fig. 4). The black solid line is the projection of field strength on the time coordinate. Here the beam arrival time is defined as the time when the electric field peak arrives.

Typical measurement results of the bunch arrival time over ten minutes during normal accelerator operation are shown in Fig. 9. The arrival timing jitter between the electron bunch and the probe laser is $285 \mathrm{fs} \mathrm{rms}$, which is composed of a random fast term and slow drift term. As we can seen in Fig. 9(a), there is an obvious periodic oscillation during the slow drift. The 220 fs slow drift term can be

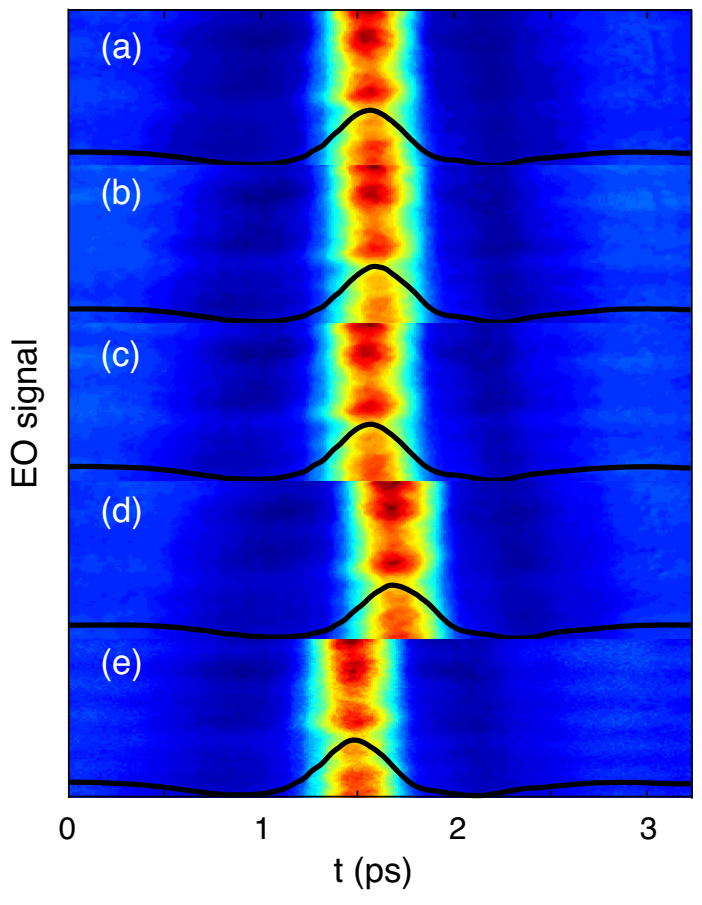

FIG. 8. (a)-(e) Single-shot EO signal of five consecutive bunches at one electric field direction (such as bottom part of Fig. 4). The black solid line is the projection of field strength on time coordinate.

attributed to the temperature of the cooling system and environment of the laser and accelerator, more details have been described in Ref. [37]. Figure 9(b) is the electron bunch arrival time without slow drift. There are several
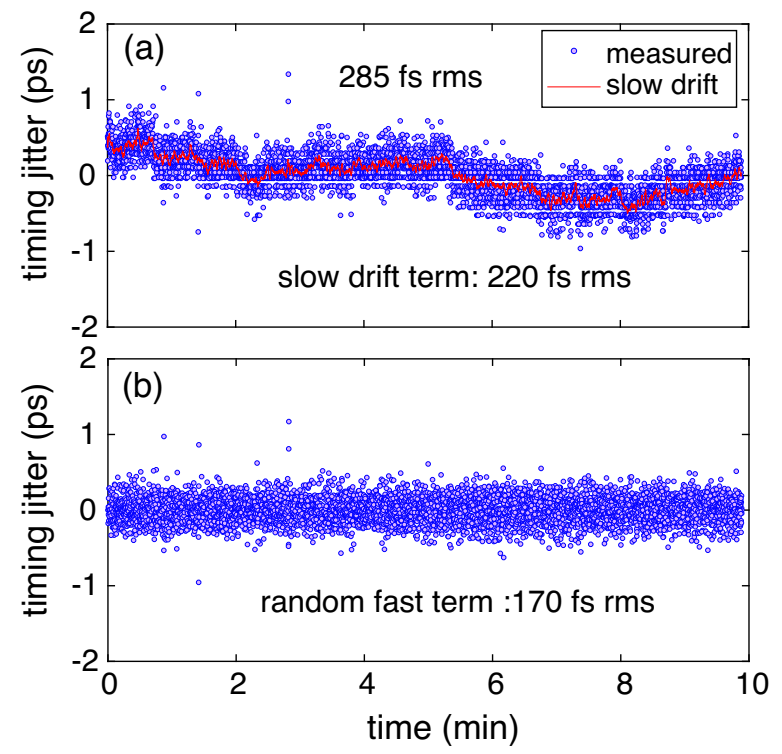

FIG. 9. The measurement of electron bunch arrival time by the EO spatial decoding technique over ten minutes. (a) The blue dots represent 6000 shots consecutive experiment date and the red line is the slow shift term. A timing jitter of $285 \mathrm{fs}$ rms is obtained from the data, in which the slow drift term timing jitter is $220 \mathrm{fs}$ rms. (b) The beam arrival time without slow drift, the corresponding fast term timing jitter is $170 \mathrm{fs}$. 
possible sources contributing to the 170 fs random fluctuation, such as the synchronization jitter of the UV driver laser and the IR probe laser with respect to the $2856 \mathrm{MHz}$ master reference signal, the fluctuations of rf phase and amplitude. Much smaller timing jitter than our previous work [37] has been achieved as a sub-100 fs timing and synchronization system was installed at TTX [58].

\section{CONCLUSION}

In conclusion, the spatial-temporal electric field distribution of radially polarized CTR THz pulses was measured with the single-shot EO spatial decoding method. Intense quasi-half-cycle transients of $\mathrm{THz}$ pulses with peak electric field up to $\sim 42 \mathrm{MV} / \mathrm{m}$ were measured at TTX with $200 \mathrm{pC}$, $30 \mathrm{MeV}$ ultrashort electron beams. A symmetrical field profile across the transverse center and the distorted wave front of CTR THz pulses were demonstrated and analyzed. By measuring and analyzing the spatial-temporal distribution of the CTR THz field at the focal plane, we provide an effective reference for the applications of CTR, such as design of coupling structures in the THz-driven electron acceleration experiment. Furthermore, the measurement of $\mathrm{THz}$ radiation by $\mathrm{EO}$ spatial decoding detection was applied to monitor the arrival time of the electron beam relative to the probe laser with femtosecond accuracy. The measured rms timing jitter is 285 fs over the ten-minute measurement, including the random fast term $(170 \mathrm{fs})$ and slow drift component (220 fs), which could be used for future beam feedback control. This single-shot method obtains the temporal waveform and 1D spatial distribution of $\mathrm{THz}$ fields simultaneously, featuring high time resolution and simple optical setup, which makes it suitable for comprehensive diagnostics of spatial-temporal waveform of $\mathrm{THz}$ pulses.

\section{ACKNOWLEDGMENTS}

This work was supported by the National Natural Science Foundation of China (NSFC Grant No. 11835004).

[1] M. Liu, H. Y. Hwang, H. Tao, A. C. Strikwerda, K. Fan, G. R. Keiser, A. J. Sternbach, K. G. West, S. Kittiwatanakul, and J. Lu, Terahertz-field-induced insulator-to-metal transition in vanadium dioxide metamaterial, Nature (London) 487, 345 (2012).

[2] B. D. O'Shea, G. Andonian, S. K. Barber, C. I. Clarke, P. D. Hoang, M. J. Hogan, B. Naranjo, O. B. Williams, V. Yakimenko, and J. B. Rosenzweig, Conductivity Induced by High-Field Terahertz Waves in Dielectric Material, Phys. Rev. Lett. 123, 134801 (2019).

[3] E. A. Nanni, W. R. Huang, K.-H. Hong, K. Ravi, A. Fallahi, G. Moriena, R. J. Dwayne Miller, and F. X. Kärtner, Terahertz-driven linear electron acceleration, Nat. Commun. 6, 8486 (2015).
[4] C. Kealhofer, W. Schneider, D. Ehberger, A. Ryabov, F. Krausz, and P. Baum, All-optical control and metrology of electron pulses, Science 352, 429 (2016).

[5] R. K. Li, M. C. Hoffmann, E. A. Nanni, S. H. Glenzer, M. E. Kozina, A. M. Lindenberg, B. K. Ofori-Okai, A. H. Reid, X. Shen et al., Terahertz-based subfemtosecond metrology of relativistic electron beams, Phys. Rev. Accel. Beams 22, 012803 (2019).

[6] D. Zhang, A. Fallahi, M. Hemmer, X. Wu, M. Fakhari, Y. Hua, H. Cankaya, A.-L. Calendron, L. E. Zapata, F. X. Kärtner et al., Segmented terahertz electron accelerator and manipulator (STEAM), Nat. Photonics 12, 336 (2018).

[7] L. Zhao, Z. Wang, C. Lu, R. Wang, C. Hu, P. Wang, L. Yan, L. Zhu, D. Xiang, J. Zhang et al., Terahertz Streaking of Few-Femtosecond Relativistic Electron Beams, Phys. Rev. X 8, 021061 (2018).

[8] J. A. Fülöp, L. Pálfalvi, M. C. Hoffmann, and J. Hebling, Towards generation of mJ-level ultrashort $\mathrm{THz}$ pulses by optical rectification, Opt. Express 19, 15090 (2011).

[9] X. Wu, J. Ma, B. Zhang, S. Chai, Z. Fang, C. Y. Xia, D. y. Kong, J. g. Wang, H. Liu, C. Q. Zhu et al., Highly efficient generation of $0.2 \mathrm{~mJ}$ terahertz pulses in lithium niobate at room temperature with sub-50 fs chirped Ti:sapphire laser pulses, Opt. Express 26, 7107 (2018).

[10] C. Vicario, B. Monoszlai, and C. P. Hauri, GV/m SingleCycle Terahertz Fields from a Laser-Driven Large-Size Partitioned Organic Crystal, Phys. Rev. Lett. 112, 213901 (2014).

[11] C. Vicario, A. V. Ovchinnikov, S. I. Ashitkov, M. B. Agranat, V.E. Fortov, and C. P. Hauri, Generation of 0.9-mJ THz pulses in DSTMS pumped by a Cr:Mg2SiO4 laser, Opt. Lett. 39, 6632 (2014).

[12] M. C. Hoffmann, S. Schulz, S. Wesch, S. Wunderlich, A. Cavalleri, and B. Schmidt, Coherent single-cycle pulses with $\mathrm{MV} / \mathrm{cm}$ field strengths from a relativistic transition radiation light source, Opt. Lett. 36, 4473 (2011).

[13] A. Perucchi, S. Di Mitri, G. Penco, E. Allaria, and S. a. Lupi, The TeraFERMI terahertz source at the seeded FERMI free-electron-laser facility, Rev. Sci. Instrum. 84, 022702 (2013).

[14] S. Casalbuoni, B. Schmidt, P. Schmüser, V. Arsov, and S. Wesch, Ultrabroadband terahertz source and beam line based on coherent transition radiation, Phys. Rev. ST Accel. Beams 12, 030705 (2009).

[15] E. Chiadroni, M. Bellaveglia, P. Calvani, M. Castellano, L. Catani, A. Cianchi, G. Di Pirro, M. Ferrario, G. Gatti, O. Limaj et al., Characterization of the $\mathrm{THz}$ radiation source at the Frascati linear accelerator, Rev. Sci. Instrum. 84, 022703 (2013).

[16] M. J. Nasse, M. Schuh, S. Naknaimueang, M. Schwarz, A. Plech, Y.-L. Mathis, R. Rossmanith, P. Wesolowski, E. Huttel, M. Schmelling et al., FLUTE: A versatile linacbased THz source, Rev. Sci. Instrum. 84, 022705 (2013).

[17] B. Green, S. Kovalev, V. Asgekar, G. Geloni, U. Lehnert, T. Golz, M. Kuntzsch, C. Bauer, J. Hauser, J. Voigtlaender et al., High-field nigh-repetition-Rate sources for the coherent THz control of matter, Sci. Rep. 6, 22256 (2016).

[18] D. Daranciang, J. Goodfellow, M. Fuchs, H. Wen, S. Ghimire, D. A. Reis, H. Loos, A. S. Fisher, and A. M. Lindenberg, Single-cycle terahertz pulses with $>0.2 \mathrm{~V} / \AA$ 
field amplitudes via coherent transition radiation, Appl. Phys. Lett. 99, 141117 (2011).

[19] Z. Wu, A. S. Fisher, J. Goodfellow, M. Fuchs, D. Daranciang, M. Hogan, H. Loos, and A. a. Lindenberg, Intense terahertz pulses from SLAC electron beams using coherent transition radiation, Rev. Sci. Instrum. 84, 022701 (2013).

[20] G. Liao, Y. Li, H. Liu, G. G. Scott, D. Neely, Y. Zhang, B. Zhu, Z. Zhang, C. Armstrong, E. Zemaityte et al., Multimillijoule coherent terahertz bursts from picosecond laser-irradiated metal foils, Proc. Natl. Acad. Sci. U.S.A. 116, 3994 (2019).

[21] A. Woldegeorgis, T. Kurihara, M. Almassarani, B. Beleites, R. Grosse, F. Ronneberger, and A. Gopal, Multi-MV/cm longitudinally polarized terahertz pulses from laserthin foil interaction, Optica 5, 1474 (2018).

[22] H. Xu, L. Yan, Y. Du, W. Huang, Q. Tian, R. Li, Y. Liang, S. Gu, J. Shi, and C. Tang, Cascaded high-gradient terahertz-driven acceleration of relativistic electron beams, arXiv:2005.11714.

[23] U. Happek, A. J. Sievers, and E. B. Blum, Observation of Coherent Transition Radiation, Phys. Rev. Lett. 67, 2962 (1991).

[24] Y. Shibata, T. Takahashi, T. Kanai, K. Ishi, M. Ikezawa, J. Ohkuma, S. Okuda, and T. Okada, Diagnostics of an electron beam of a linear accelerator using coherent transition radiation, Phys. Rev. E 50, 1479 (1994).

[25] D. Mihalcea, C. L. Bohn, U. Happek, and P. Piot, Longitudinal electron bunch diagnostics using coherent transition radiation, Phys. Rev. ST Accel. Beams 9, 082801 (2006).

[26] I. Nozawa, K. Kan, J. Yang, A. Ogata, T. Kondoh, M. Gohdo, K. Norizawa, H. Kobayashi, H. Shibata, S. Gonda et al., Measurement of $20 \mathrm{fs}$ bunch length using coherent transition radiation, Phys. Rev. ST Accel. Beams 17, 072803 (2014).

[27] T. Y. Zhou, Y. L. Yang, B. G. Sun, L. L. Tang, P. Lu, Z. R. Zhou, F. F. Wu, and X. Y. Liu, Longitudinal electron bunch diagnostics using coherent transition radiation at the IRFEL, in Proceedings of the 8th International Symposium on Advanced Optical Manufacturing and Testing Technologies: Optical Test, Measurement Technology, and Equipment (International Society for Optics and Photonics, Suzhou, China, 2016), Vol. 9684, p. 968411.

[28] J. van Tilborg, C. B. Schroeder, C. V. Filip, C. Tóth, C. G. R. Geddes, G. Fubiani, R. Huber, R. A. Kaindl, E. Esarey, and W. P. Leemans, Temporal Characterization of Femtosecond Laser-Plasma-Accelerated Electron Bunches using Terahertz Radiation, Phys. Rev. Lett. 96, 014801 (2006).

[29] A. D. Debus, M. Bussmann, U. Schramm, R. Sauerbrey, C. D. Murphy, Z. Major, R. Hörlein, L. Veisz, K. Schmid, J. Schreiber et al., Electron Bunch Length Measurements from Laser-Accelerated Electrons using Single-Shot THz Time-Domain Interferometry, Phys. Rev. Lett. 104, 084802 (2010).

[30] Z. Zhang, L. Yan, Y. Du, Z. Zhou, X. Su, L. Zheng, D. Wang, Q. Tian, W. Wang, J. Shi, H. Chen, W. Huang, W. Gai, and C. Tang, Tunable High-Intensity Electron Bunch Train Production Based on Nonlinear Longitudinal Space Charge Oscillation, Phys. Rev. Lett. 116, 184801 (2016).
[31] M. Chen, J.-C. Deinert, B. Green, Z. Wang, I. Ilyakov, N. Awari, M. Bawatna, S. Germanskiy, T. V. A. G. de Oliveira, G. Geloni, T. Tanikawa, M. Gensch, and S. Kovalev, Pulse and field-resolved $\mathrm{THz}$ diagnostics at 4th generation light sources, Opt. Express 27, 32360 (2019).

[32] G. Berden, A. F. Van Der Meer, S. P. Jamison, B. Steffen, E. A. Knabbe, B. Schmidt, P. Schmüser, A. M. MacLeod, P. J. Phillips, and W. A. Gillespie, Time resolved singleshot measurements of transition radiation at the $\mathrm{THz}$ beam line of FLASH using electro-optic spectral decoding, in Proceedings of the 10th European Particle Accelerator Conference, Edinburgh, Scotland, 2006 (EPS-AG, Edinburgh, Scotland, 2006), Vol. 1, p. 1058.

[33] K. Huang, H. Kotaki, M. Mori, T. Esirkepov, J. K. Koga, Y. Hayashi, N. Nakanii, S. V. Bulanov, and M. Kando, Variation in electron emission time in weakly nonlinear laser wakefield acceleration, Phys. Rev. Accel. Beams 22, 121301 (2019).

[34] I. Wilke, A. M. MacLeod, W. A. Gillespie, G. Berden, G. M. H. Knippels, and A. F. G. van der Meer, Single-Shot Electron-Beam Bunch Length Measurements, Phys. Rev. Lett. 88, 124801 (2002).

[35] G. Berden, S. P. Jamison, A. M. MacLeod, W. A. Gillespie, B. Redlich, and A.F.G. van der Meer, Electro-Optic Technique with Improved Time Resolution for Real-Time, Nondestructive, Single-Shot Measurements of Femtosecond Electron Bunch Profiles, Phys. Rev. Lett. 93, 114802 (2004).

[36] A. L. Cavalieri, D. M. Fritz, S. H. Lee, P. H. Bucksbaum, D. A. Reis, J. Rudati, D. M. Mills, P. H. Fuoss, G. B. Stephenson, C. C. Kao, and D. P. Siddons, Clocking Femtosecond X Rays, Phys. Rev. Lett. 94, 114801 (2005).

[37] W. Wang, Y. Du, L. Yan, Z. Chi, Z. Zhang, J. Hua, W. Huang, C. Tang, and M. Li, Temporal profile monitor based on electro-optic spatial decoding for low-energy bunches, Phys. Rev. Accel. Beams 20, 112801 (2017).

[38] K. Huang, T. Esirkepov, J. K. Koga, H. Kotaki, M. Mori, Y. Hayashi, N. Nakanii, S. V. Bulanov, and M. Kando, Electro-optic spatial decoding on the spherical-wave-front Coulomb fields of plasma electron sources, Sci. Rep. 8, 2938 (2018).

[39] B. Steffen, V. Arsov, G. Berden, W. A. Gillespie, S. P. Jamison, A. M. MacLeod, A. F. G. van der Meer, P. J. Phillips, H. Schlarb, B. Schmidt, and P. Schmüser, Electrooptic time profile monitors for femtosecond electron bunches at the soft x-ray free-electron laser flash, Phys. Rev. ST Accel. Beams 12, 032802 (2009).

[40] S. P. Jamison, G. Berden, A. M. MacLeod, D. A. Jaroszynski, B. Redlich, A. F. Van Der Meer, and W. A. Gillespie, Electro-optic techniques for temporal profile characterization of relativistic Coulomb fields and coherent synchrotron radiation, Nucl. Instrum. Methods Phys. Res., Sect. A 557, 305 (2006).

[41] C. Szwaj, C. Evain, M. Le Parquier, P. Roy, L. Manceron, J.-B. Brubach, M.-A. Tordeux, and S. a. Bielawski, High sensitivity photonic time-stretch electro-optic sampling of terahertz pulses, Rev. Sci. Instrum. 87, 103111 (2016).

[42] S. Casalbuoni, B. Schmidt, P. Schmuser, and B. Steffen, Far-infrared transition and diffraction radiation part I: Production, diffraction effects and optical propagation, Tesla-Report No. 15, 2005, p. 1. 
[43] J. R. Fletcher, Distortion and uncertainty in chirped pulse THz spectrometers, Opt. Express 10, 1425 (2002).

[44] J. van Tilborg, C. B. Schroeder, C. Tóth, C. G. R. Geddes, E. Esarey, and W. P. Leemans, Single-shot spatiotemporal measurements of high-field terahertz pulses, Opt. Lett. 32, 313 (2007)

[45] N. H. Matlis, G. R. Plateau, J. van Tilborg, and W. P. Leemans, Single-shot spatiotemporal measurements of ultrashort $\mathrm{THz}$ waveforms using temporal electricfield cross correlation, J. Opt. Soc. Am. B 28, 23 (2011).

[46] Y. Du, L. Yan, J. Hua, Q. Du, Z. Zhang, R. Li, H. Qian, W. Huang, H. Chen, and C. Tang, Generation of first hard $\mathrm{x}$-ray pulse at Tsinghua Thomson scattering x-ray source, Rev. Sci. Instrum. 84, 053301 (2013).

[47] D. Xiang and W. H. Huang, Properties of diffraction radiation in practical conditions: Finite size target effect, surface roughness and prewave zone, Nucl. Instrum. Methods Phys. Res., Sect. B 248, 163 (2006).

[48] D. Xiang and W.-H. Huang, Investigations on collective behavior in radiation process with random walk model, Nucl. Instrum. Methods Phys. Res., Sect. B 240, 855 (2005).

[49] S. Casalbuoni, H. Schlarb, B. Schmidt, P. Schmüser, B. Steffen, and A. Winter, Numerical studies on the electrooptic detection of femtosecond electron bunches, Phys. Rev. ST Accel. Beams 11, 072802 (2008).

[50] G. Gallot, J. Zhang, R. W. Mcgowan, T.-I. Jeon, and D. a. Grischkowsky, Measurements of the $\mathrm{THz}$ absorption and dispersion of $\mathrm{ZnTe}$ and their relevance to the electrooptic detection of $\mathrm{THz}$ radiation, Appl. Phys. Lett. 74, 3450 (1999).
[51] J. Shan, A. S. Weling, E. Knoesel, L. Bartels, M. Bonn, A. Nahata, G. A. Reider, and T. F. Heinz, Single-shot measurement of terahertz electromagnetic pulses by use of electro-optic sampling, Opt. Lett. 25, 426 (2000).

[52] L. Yan, J. Hua, Y. Du, Y. Huang, Y. You, D. Wang, W. Huang, and C. Tang, UV pulse trains by $\alpha$-BBO crystal stacking for the production of $\mathrm{THz}$ rap-rate electron bunches, J. Plasma Phys. 78, 429 (2012).

[53] C. Tang, W. Huang, R. Li, Y. Du, L. Yan, J. Shi, Q. Du, P. Yu, H. Chen, T. Du et al., Tsinghua Thomson scattering X-ray source, Nucl. Instrum. Methods Phys. Res., Sect. A 608, S70 (2009).

[54] G. Sharma, K. Singh, I. Al-Naib, R. Morandotti, and T. Ozaki, Terahertz detection using spectral domain interferometry, Opt. Lett. 37, 4338 (2012).

[55] Z. Jiang, F. G. Sun, Q. Chen, and X. C. Zhang, Electrooptic sampling near zero optical transmission point, Appl. Phys. Lett. 74, 1191 (1999).

[56] J. van Tilborg, C. B. Schroeder, E. Esarey, and W. P. Leemans, Pulse shape and spectrum of coherent diffraction-limited transition radiation from electron beams, Laser Part. Beams 22, 415 (2004).

[57] Y. Wang, L. Yan, Z. Zhao, and Z. Chen, Absolute energy measurement of single-shot terahertz pulse with a thermal detector, Laser and Tera-Hertz Science and Technology, LTST 2012, STh4A.19 (2012), https://doi.org/10.1364/ LTST.2012.STh4A.19.

[58] Z. Lin, Y. Du, J. Yang, Y. Xu, L. Yan, W. Huang, C. Tang, G. Huang, Q. Du, L. Doolittle et al., Development of sub100 femtosecond timing and synchronization system, Rev. Sci. Instrum. 89, 014701 (2018). 